\title{
Preceptoria nos serviços públicos especializados como cenário de aprendizagem na formação em Odontologia
}

\author{
Rebeca Valeska Soares Pereira*; Lydiane dos Santos Dantas*; Ítalo de Macedo Bernardino**; Viviane \\ Costa Silva***; Renata Cardoso Rocha Madruga****; Rilva Suely de Castro Cardoso Lucas**** \\ * Graduada em Odontologia pela Universidade Estadual da Paraíba \\ ** Mestrando em Clínicas Odontológicas, Universidade Estadual da \\ Paraíba \\ *** Graduanda em Odontologia, Universidade Estadual da Paraíba \\ **** Professora Doutora, Curso de Odontologia, Universidade Estadual \\ da Paraíba
}

Recebido em 27/03/2018. Aprovado em 21/12/2018.

\begin{abstract}
RESUMO
A preceptoria é um dos pilares no processo da integração ensino-serviço, oportunizando a prática de diversas competências profissionais e propondo uma nova forma de pensar a formação. Partilhar experiências, articular teoria e prática de forma dinâmica e participativa, incentivar o estudante induzindo-o a uma abordagem integral do paciente e comprometimento com a equipe de saúde, o serviço e o SUS, são práticas que proporcionam ganhos no processo de aprendizagem. Esta pesquisa objetivou traçar o perfil de competências e habilidades dos cirurgiões-dentistas que atuam nos serviços públicos de atenção secundária e terciária à saúde na cidade de Campina Grande/PB a fim de identificar as características e expectativas acerca do exercício da preceptoria. Tratou-se de uma pesquisa observacional, quantitativa, descritiva, do tipo transversal, desenvolvida por meio de pesquisa de campo, com levantamento de dados por meio de aplicação de questionário. A maioria dos profissionais tinha até 37 anos de idade $(55,9 \%)$, sexo masculino $(52,9 \%)$, com tempo de formação igual ou inferior há 13 anos, assinalando que a integração ensino-serviço é extremamente importante $(70,6 \%)$, nunca participou de formação para preceptores $(67,6 \%)$. Todos os profissionais com menor tempo de formação relataram sentir-se preparados para a função de preceptoria e, nesse aspecto, a maioria que desejou atuar como preceptor $(92,0 \%)$ afirmou sentir-se preparado para a função. Os participantes compreendem a integração ensino-serviço como potencial estratégia colaboradora do processo de mudança de práticas na formação em saúde, demonstrando desejo de atuar na preceptoria e participar de programas de capacitação em educação permanente.
\end{abstract}

Descritores: Preceptoria. Saúde Pública. Ensino. Odontologia. 


\section{INTRODUÇÃO}

De acordo com a Constituição Federal, a gestão do Sistema Único de Saúde (SUS) é responsável pelo ordenamento da formação de recursos humanos da área da saúde, bem como o incremento na sua área de atuação, do desenvolvimento científico e tecnológico. $\mathrm{O}$ artigo 27 da lei 8080/90 ${ }^{1}$ legitima que os serviços públicos que integram o SUS são ambientes de prática para o ensino e a pesquisa, propiciando a articulação dos interesses das Instituições de Educação Superior e do SUS, a fim de melhorar a qualidade do serviço prestado à população ${ }^{2}$.

As Diretrizes Curriculares Nacionais (DCN) para os cursos de graduação em saúde implementadas a partir de $2002^{3}$, reafirmaram a importância e o dever da formação voltada às necessidades do SUS para profissionais de saúde, a fim de adequar a atuação profissional à realidade da população brasileira e proporcionar aos estudantes a aplicação dos conhecimentos teóricos, não apenas de forma técnica, mas desenvolver habilidades humanizadas, críticas e relacionais ${ }^{4,5}$.

Dentre as importantes vertentes presentes nesta reestruturação está a integração ensinoserviço, compreendida como eixo fundamental do processo pedagógico, é responsável pela integração da universidade aos serviços de saúde concedendo privilégios ao estudante no que se diz respeito à obtenção de conhecimentos, expectativas e experiências relativos ao processo formativo ${ }^{6}$. O formato diferenciado de atenção à saúde e o sistema em rede reflete nas experiências de estágio, proporcionando a vivência da realidade nos níveis de atenção, além das experiências de gestão, promovendo a compreensão sobre as conformações das Redes de Atenção em Saúde ${ }^{7,8}$.

Em tais cenários de aprendizagem, a grande característica do preceptor em saúde é ele ser o profissional que atua dentro do ambiente de assistência à saúde e que também o torna propício para o ensino e para a prática profissional. A intermediação da formação, o desenvolvimento de habilidades clínicas e rotineiras, e a avaliação do profissional em formação, mostram-se como funções primordiais ${ }^{9,10}$.

O desafio contínuo de formar profissionais de saúde com consciência das necessidades da população e com habilidades essenciais para o desenvolvimento das suas atividades mostra-se presente no dia a dia da formação e vivência, tanto daqueles que já atuam no SUS, quanto dos que estão na graduação. Observa-se que na literatura ainda há uma escassez de estudos que visam compreender o perfil de competências dos profissionais da área de Odontologia, atuantes no SUS, no tocante ao exercício da preceptoria, dessa forma, demonstra-se a relevância desses dados para a integração ensino-serviço e à própria gestão tomar ciência do perfil de competências dos profissionais que integram sua rede de assistência.

Neste sentido, o objetivo deste estudo foi elaborar e aplicar um instrumento de avaliação do perfil de competências do cirurgião-Dentista investido no papel de preceptor nos níveis de média e alta complexidade no SUS do Município de Campina Grande/PB.

\section{METODOLOGIA}

Trata-se de um estudo transversal e exploratório, quantitativo e analítico, com um desenho do tipo transversal. A pesquisa foi desenvolvida na cidade de Campina Grande/PB, região Nordeste do Brasil, com todos os profissionais da área de Odontologia atuantes nos níveis de média e alta complexidade no SUS.

No nível de média complexidade foram abordados os profissionais em exercício nos Centros de Especialidades Odontológicas (CEOs) e no Hospital Universitário Alcides Carneiro (HUAC), totalizando 21 cirurgiões-dentistas. $\mathrm{Na}$ atenção terciária, representada pelo Hospital de Emergência e Trauma Dom Luiz Gonzaga Fernandes, atuavam 14 cirurgiões bucomaxilo- 
faciais plantonistas. Foi excluído um profissional que não se encontrava em exercício pleno da função durante o período da pesquisa.

O questionário utilizado para avaliar o perfil de competências do preceptor e respectivos campos de trabalho foi construído a partir das DCN para os cursos de Odontologia, aprovadas em 2002, com o intuito de adequar a formação na área de maneira coerente com as necessidades do SUS, as quais proporcionam as bases para a reorientação do modelo de ensino ${ }^{3}$.

Os dados obtidos foram submetidos à análise estatística descritiva objetivando caracterizar a amostra, bem como analítica, em que foi empregado o teste exato de Fisher para investigar associações entre as variáveis qualitativas estudadas ${ }^{11}$. O nível de significância foi fixado em $5 \%(\mathrm{p}<0,05)$. Todas as análises foram feitas usando o software IBM SPSS Statistics versão 20.0, considerando um intervalo de confiança de $95 \%$.

$\mathrm{O}$ estudo obedeceu às normas da Resolução № 466/2012 do Conselho Nacional de Saúde que regulamenta a pesquisa em seres humanos, obtendo parecer aprovado do Comitê de Ética em Pesquisa da Universidade Estadual da Paraíba, sob o número do CAAE: 63009916.7.0000.5187, recebendo também anuência das respectivas instituições participantes do estudo.

\section{RESULTADOS E DISCUSSÃO}

A tabela 1 mostra a distribuição dos participantes de acordo com as características sociodemográficas, perfil de formação e atuação profissional. A maioria tinha até 37 anos de idade, era do sexo masculino, relatou ter se formado há 13 anos ou menos e atuava na atenção secundária.

Tabela 1. Distribuição dos participantes de acordo com as características sociodemográficas, tempo de formação e atuação profissional

\begin{tabular}{lcc}
\hline Variáveis & n & $\%$ \\
\hline Idade* $^{*}$ & & \\
$\leq 37$ anos & 19 & 55,9 \\
$>37$ anos & 15 & 44,1 \\
Sexo & & \\
$\quad$ Masculino & 18 & 52,9 \\
Feminino & 16 & 47,1 \\
Tempo de formação (em anos)* & & \\
$\leq 13$ anos & 19 & 55,9 \\
$>13$ anos & 15 & 44,1 \\
Nível de atenção & & \\
Secundária & 20 & 58,8 \\
Terciária & 14 & 41,2 \\
Tempo de atuação no serviço (em anos)* & & \\
$\leq 7$ anos & 20 & 58,8 \\
$>7$ anos & 14 & 41,2 \\
\hline
\end{tabular}

* Variáveis dicotomizadas pela mediana

Assim como identificado no presente cirurgião-dentista (tabela 2), dentre os estudo, outros autores ${ }^{12-14}$ destacaram a diferenciais de tal integração pode-se destacar a integração do ensino-serviço como um fator correlação teórico-prática, o trabalho em equipe, extremamente importante na formação do a reorganização do modelo de cuidado e o 
enfrentamento das verdadeiras necessidades em saúde da população.

Tabela 2. Distribuição dos participantes de acordo com as percepções sobre a integração ensinoserviço

\begin{tabular}{|c|c|c|}
\hline Variáveis & $\mathbf{n}$ & $\%$ \\
\hline \multicolumn{3}{|c|}{$\begin{array}{l}\text { Como você avalia a integração ensino-serviço (estagiários na rede do SUS) } \\
\text { na formação do cirurgião-dentista? }\end{array}$} \\
\hline Sem importância & 0 & 0,0 \\
\hline Pouco importante & 0 & 0,0 \\
\hline Importante & 6 & 17,6 \\
\hline Muito importante & 4 & 11,8 \\
\hline Extremamente importante & 24 & 70,6 \\
\hline \multicolumn{3}{|c|}{ Você tem conhecimento do significado da preceptoria na Odontologia? } \\
\hline Sim & 33 & 97,1 \\
\hline Não & 1 & 2,9 \\
\hline \multicolumn{3}{|c|}{$\begin{array}{l}\text { Você acredita que a atividade de preceptoria realmente é importante na } \\
\text { formação do aluno? }\end{array}$} \\
\hline $\operatorname{Sim}$ & 34 & 100,0 \\
\hline Não & 0 & 0,0 \\
\hline \multicolumn{3}{|c|}{ Você já participou de alguma formação para preceptores? } \\
\hline Sim & 11 & 32,4 \\
\hline Não & 23 & 67,6 \\
\hline
\end{tabular}

As iniciativas propiciadas por tal integração fortalecem a corresponsabilização das instituições envolvidas no processo, a universidade na prestação e potencialização do cuidado e os serviços na atuação junto à formação e envolvimento dos preceptores ${ }^{12,13,15}$. O estágio proporciona um contato efetivo entre o estudante $\mathrm{e}$ a comunidade, extremamente produtivo para o processo de ensino-aprendizagem, e oportuniza o desenvolvimento de relações, aguçando a percepção do indivíduo, que inserido no meio social tem uma formação mais humanizada em saúde ${ }^{12,16-18}$.

A formação didático-pedagógica dos preceptores é necessária para interação adequada com os estudantes nos serviços públicos de saúde ${ }^{6}$. Ponto chave e de extrema relevância, a ausência de programas de capacitação direcionados ao profissional para o exercício da preceptoria, é identificado a partir dos resultados obtidos (tabela
2), e relatado na literatura que os preceptores não possuem a formação continuada como item presente na agenda de trabalho ${ }^{6,19}$.

Autores $^{20,21}$ destacam a importância da atualização dos profissionais de saúde devendo ser realizada de forma permanente e continuada, não fragmentada, fazendo parte do dia a dia, contribuindo no desempenho da sua função, promovendo uma reflexão consistente sobre o modelo de atenção à saúde a partir de parcerias estabelecidas entre as instituições de ensino, os serviços, a comunidade, as entidades e os setores da sociedade civil que possam ser envolvidos.

A tabela 3 mostra a distribuição dos participantes de acordo com as percepções sobre a atividade de preceptoria e a relação com o estagiário. A maioria relatou que atua ou já atuou como preceptor e que existem dificuldades no processo de desenvolvimento da preceptoria. As três dificuldades mais apontadas foram: falta 
programa de capacitação para atuar na preceptoria em saúde, falta de incentivo financeiro e falta de apoio da instituição de onde os estudantes advêm.

Dentro desse contexto, a falta de programas de capacitação para atuação na preceptoria obteve destaque como uma das principais dificuldades enfrentadas para o exercício da mesma, característica também presente no estudo realizado por Souza e Carcereri 6 .

A falta de incentivo financeiro para o desempenho da preceptoria e a falta de apoio da instituição de ensino foram outros aspectos que obtiveram destaque na listagem das dificuldades enfrentadas. Dificuldades estas também encontradas de forma coerente no estudo desenvolvido por Trajman et $a .^{22}$, em que a maioria dos profissionais da rede não encontra apoio institucional ou oferta de oportunidades para acesso a cursos de formação especializada em saúde da família, em saúde coletiva ou mesmo para o exercício de uma clínica ampliada de cunho generalista, conforme as prioridades estabelecidas para o setor.

Ainda em conformidade com o estudo, expõe-se a necessidade da melhoria das condições de trabalho no que se diz respeito à remuneração. Trajman et $a l^{22}$ ressaltam que a melhor remuneração por suas atividades é mais importante do que o complemento salarial para a tarefa de preceptoria. Desta forma, compreende-se que o profissional entende a preceptoria como parte de suas atribuições, podendo ser incorporadas ao ambiente de trabalho como parte da estratégia de educação permanente.

Missaka e Ribeiro ${ }^{20}$ afirmam que sem a devida capacitação e participação do preceptor no que se diz respeito ao planejamento e execução das atividades voltadas para o estágio, os objetivos, eficácia e resultados deste, provavelmente, não serão alcançados. Salienta-se que a formação dos preceptores deve ser apoiada e tida como função prioritária das Instituições de Ensino Superior, voltada tanto para à atualização profissional quanto às funções de ensino. Dentre as funções atribuídas às universidades, essa é uma das suas grandes vocações, devendo ser a principal contrapartida nos convênios com as Secretarias Municipais de Saúde ${ }^{22}$.

Tabela 3. Distribuição dos participantes de acordo com as percepções sobre a atividade de preceptoria

\begin{tabular}{lcc}
\multicolumn{1}{c}{ Variáveis } & n & \% \\
\hline Você atua ou já atuou como preceptor? & 24 & 70,6 \\
Sim & 10 & 29,4 \\
Não & & \\
Existem dificuldades no processo de desenvolvimento da preceptoria? & 23 & 67,6 \\
Sim & 11 & 32,4 \\
Não & & \\
Das alternativas a seguir, quais você considera como dificuldade para o & & \\
exercício da preceptoria?* & 24 & 70,6 \\
Falta programa de capacitação para atuar na preceptoria em saúde & 12 & 35,3 \\
Falta comprometimento dos estudantes & 1 & 2,9 \\
Desperdício de tempo clínico com orientação aos alunos & 1 & 2,9 \\
Demanda alta de alunos & 6 & 17,6 \\
Aumento do volume de trabalho & 20 & 58,8 \\
Falta de incentivo financeiro & 18 & 52,9 \\
Falta de apoio da instituição de onde os estudantes advêm & 14 & 41,2 \\
Falta de insumos (instrumentais, EPI's, materiais educativos, etc) & &
\end{tabular}

* O participante podia assinalar mais de uma alternativa. 
As competências e habilidades pautadas no instrumento de estudo, estão intimamente ligadas aos conhecimentos requeridos para a formação do cirurgião dentista de acordo com o artigo $4^{\circ}$ das DCN que são Atenção à saúde, Tomada de decisões, Comunicação, Liderança, Administração e gerenciamento, Educação permanente ${ }^{3}$.

Neste sentido, como pode ser observado na tabela 4, o respeito aos princípios da bioética e ética profissional, a atualização constante, entendendo a importância de novos conhecimentos e dos estágios para as futuras gerações de profissionais, bem como a habilidade para tomada de decisões de forma eficaz e sensata foram as competências melhor avaliadas quanto ao seu nível de importância pelos participantes do estudo. Os achados de Rocha e Ribeiro ${ }^{23}$ corroboram que dentre as características mais importantes de um bom preceptor estão o compromisso com a aprendizagem do aluno, o conhecimento do papel do preceptor como um formador e a capacidade de incentivar o aluno em sua aprendizagem.

$\mathrm{Na}$ associação dos questionamentos referentes ao tempo de formação do profissional e quanto a sentir-se preparado para a função de preceptoria (tabela 5), notou-se significância estatística. Os profissionais que são formados há treze anos ou menos em sua totalidade relataram sentir-se preparados para a função de preceptoria, ao passo que entre os profissionais com maior tempo de formação uma quantidade expressiva destacou que não se sentiam preparados para tal função. Dessa forma, percebe-se o importante papel que as DCN exercem na reestruturação curricular, desde a sua publicação e, o reflexo nas mudanças de práticas que contribuem para a integração ensino-serviço por meio das ações integradas proporcionando benefícios para ambas às partes.

A partir de tal constatação pode-se deduzir o impacto que a implantação das DCN vem causando na formação dos novos profissionais que passam pela academia e se deparam com uma nova realidade onde é defendida uma prática profissional ampla, contextualizada, com atividades "extramuros" que permitem uma aproximação das situações reais de trabalho e o desenvolvimento de competências para tal ${ }^{8,21}$.

Dessa forma, pode-se relacionar que o sentimento dos profissionais quanto a estarem preparados para o exercício da preceptoria reflete no desejo que estes apresentaram em atuar ou não nesta função. Foi identificado que a maioria dos profissionais que demonstraram o desejo de atuar como preceptor, afirmou sentir-se preparado para a função de preceptoria (tabela 6).

Ressalta-se a necessidade da criação de programas de capacitação que contribuam na formação e na educação permanente desses profissionais que se deparam no dia a dia com a necessidade de atualização constante e de medidas resolutivas em meio às circunstâncias desafiadoras, através do entendimento de práticas pedagógicas ativas favorecendo o processo de desenvolvimento do estudante que futuramente estará inserido no serviço.

Desta maneira, estabelece-se a problemática que os preceptores dominam sim, os saberes profissionais, típico para a formação, passando a impressão de estar preparados para reproduzir o conhecimento. Entretanto, não há o domínio dos saberes pedagógicos, necessários à organização de ações formativas, tais como os diversos processos de ensino-aprendizagem e as diferentes modalidades de avaliação gerando uma atuação intuitiva, podendo haver a confusão entre transmissão de informação com ensino ${ }^{20}$.

\section{CONSIDERAÇÕES FINAIS}

Após análise dos dados coletados, o estudo apontou que nos níveis de média e alta complexidade, entre os 34 profissionais, a integração ensino-serviço foi considerada extremamente importante, no entanto, uma parcela 
Tabela 4. Distribuição dos participantes de acordo com as percepções sobre as competências que caracterizam um bom perfil de preceptor

\section{Variáveis}

$\mathbf{n}$

$\%$

Avalie as competências a seguir de acordo com o grau de relevância para um bom perfil de um preceptor:

- Aptidão de desenvolvimento de atribuições em seu nível de saúde, tanto no âmbito individual quanto coletivo

Sem importância

Pouco importante

Importante

Muito importante

Extremamente importante

- Prática de forma integrada, resolutiva e contínua com as demais instâncias de saúde

Sem importância

Pouco importante

Importante

Muito importante

Extremamente importante

- Habilidade para tomada de decisões de forma eficaz e sensata

Sem importância

25,0

Pouco importante

0,0

Importante

Muito importante

Extremamente importante

- Respeito aos princípios da bioética e ética profissional

Sem importância

Pouco importante

Importante

Muito importante

Extremamente importante

- Acessibilidade, garantindo a interação com outros profissionais e o público em geral

Sem importância

0,0

$1-4,2$

14,2

$4 \quad 16,7$

$18 \quad 75,0$

Pouco importante

Importante

Muito importante

Extremamente importante

- Capacidade de liderar, administrar e gerenciar tanto os recursos humanos, quanto os recursos materiais e de informação

Sem importância

Pouco importante

Importante

Muito importante

Extremamente importante

- Atualização constante, entendendo a importância de novos conhecimentos e dos estágios para as futuras gerações de profissionais

Sem importância

Pouco importante 
Tabela 5. Associação entre tempo de formação do profissional e sentir-se preparado para a função de preceptoria

\begin{tabular}{lcccc}
\multirow{2}{*}{ Variável } & \multicolumn{3}{c}{ Tempo de formação } \\
\cline { 2 - 4 } & $\leq \mathbf{1 3}$ anos & $\mathbf{> 1 3}$ anos & Total & p-valor \\
\cline { 2 - 5 } & $\mathbf{n}(\mathbf{\%})$ & $\mathbf{n}(\boldsymbol{\%})$ & $\mathbf{n}(\boldsymbol{\%})$ & \\
\hline Você sente-se preparado para a função de preceptoria? & & & $0,004^{*}$ \\
Sim & $19(100,0)$ & $9(60,0)$ & $28(82,4)$ & \\
Não & $0(0,0)$ & $6(40,0)$ & $6(17,6)$ & \\
\hline
\end{tabular}

Teste exato de Fisher. ${ }^{*} \mathrm{p}<0,05$.

Tabela 6. Associação entre desejo de atuar como preceptor, sentir-se preparado para a função de preceptoria

Variável

Você tem desejo de atuar como

preceptor?

\begin{tabular}{cccc}
\hline Sim & Não & Total & p-valor \\
\hline n $(\%)$ & n $(\%)$ & n (\%) & \\
\hline
\end{tabular}

Você sente-se preparado para a função de preceptoria?

$\begin{array}{lrrr}\text { Sim } & 23(92,0) & 5(55,6) & 28(82,4) \\ \text { Não } & 2(8,0) & 4(44,4) & 6(17,6)\end{array}$

Teste exato de Fisher. * $\mathrm{p}<0,05$.

expressiva dos profissionais não participou de formação para preceptores, mesmo relatando que atuam ou já atuaram como preceptores e alegando dificuldades no processo da preceptoria. Houve associação significativa entre sentir-se preparado para a função de preceptor e o tempo de formação, assim como, com o desejo de atuar como preceptor.

De acordo com as constatações do estudo, sugere-se que para a obtenção de melhor efetividade na evolução dos processos de aprendizagem pactuados a partir das necessidades e expectativas dos profissionais, impende-se a realização de estudos semelhantes com outras categorias profissionais da saúde, abrangendo localidades polarizadas pelos municípios que ofertam formação superior em saúde, avaliando os contingentes e perfis de preceptores, considerando as recomendações dos órgãos oficiais que regem o ensino superior no país.

\section{ABSTRACT \\ Preceptory practice in specialized public services as a learning scenario in dentistry training}

The preceptory practice is one of the pillars in the process of teaching-service integration, allowing the practice of various professional skills and proposing a new way of thinking about training. Sharing experiences, bringing together theory and practice in a dynamic and participative way, leading the student to perceive the integral approach of the patient and commitment to the health team, the SUS service (Brazilian Unified Health Service), are practices that provide gains in the learning process. This research aimed to outline the profile of skills and abilities of dentists who work in public secondary and tertiary health care services in the city of Campina Grande / PB in order to identify the characteristics and expectations about the preceptory practice. This is an observational, quantitative, descriptive, cross-sectional study 
developed through field research with data collection through questionnaire application. The majority of professionals aged up to 37 years $(55.9 \%)$, males $(52.9 \%)$, with graduation time of 13 years or less, who reported that teachingservice integration is extremely important $(70.6 \%)$, or never participated in training for preceptors (67.6\%). All professionals with shorter graduation time reported feeling prepared for the preceptory function and, in this aspect, the majority who wanted to act as preceptor $(92.0 \%)$ reported that they felt prepared for the activity. Participants understand the teaching-service integration as a potential collaborative strategy of the process of changing practices in health education, demonstrating the desire to work as preceptors and participate in training programs in lifelong education.

Descriptors: Preceptorship. Public Health. Teaching. Dentistry.

\section{REFERÊNCIAS}

1. Brasil. Lei n ${ }^{\circ} 8080$, de 19 de setembro de 1990. Dispõe sobre as condições para a promoção, proteção e recuperação da saúde, a organização e o funcionamento dos serviços correspondentes e dá outras providências. Diário Oficial da União, Brasília, DF, 20 de setembro de 1990. Seção 1. [Acesso em 15 de maio de 2017]. Disponível em: http://www. planalto.gov.br/ccivil_03/leis/18080.htm.

2. Cavalheiro MTP, Guimarães AL. Formação para o SUS e os desafios da integração ensino serviço. Caderno FNEPAS. 2011;1:19-27.

3. Brasil. Ministério da Educação. Resolução $n^{\circ}$ CNE/CES 3/2002 de 19 de fevereiro de 2002. Institui as Diretrizes Curriculares Nacionais dos Cursos de Farmácia e Odontologia. Diário Oficial, Brasília, DF, 04 de março de 2002, seção 1, p. 10. [Acesso em 15 de maio de 2017]. Disponível em: http://portal.mec.gov. br/cne/arquivos/pdf/CES032002.pdf.

4. Morita MC, Kriger L. Mudanças nos cursos de Odontologia e a interação com o SUS. Rev ABENO. 2004;4(1):17-21.
5. Chiesa AM, Nascimento DDG, Braccialli LAD, Oliveira MAC, Ciampone MHT. A formação de profisssionais da saúde: aprendizagem significativa à luz da promoção da saúde. Cogitare Enferm. 2007;12(2):23640.

6. Souza AL, Carcereri DL. Qualitative study of the teaching-service integration in an undergraduate Dentistry course. Interface Comunic Saúde Educ. 2011;15(39):1071-84.

7. Toassi RFC, Baumgarten A, Warmling CM, Rossoni E, Rosa AR, Slavutzky SMB. Teaching at primary health care services within the Brazilian national health system (SUS) in Brazilian health care professionals' training. Interface. 2013;17(45):385-392.

8. Warmling CM, Baldisserotto J, Stocker J, Gallo DB, Pezzato LM, Hugo FN. O agir em competência para o cuidado especializado na saúde bucal. Rev ABENO. 2015;15(1):1-16.

9. Botti SHO, Rego S. Preceptor, supervisor, tutor e mentor: quais são seus papéis?. Rev Bras Educ Méd. 2008;32(2):363-72.

10. Rocha HC, Ribeiro VB. Training course for teaching preceptors of medical internship. Rev Bras Educ Méd. 2012;36(3):343-50.

11. Larson R, Farber B. Estatística aplicada. 6. ed. São Paulo: Pearson Prentice Hall; 2016.

12. Araújo ME, Zilbovicius C. A formação acadêmica para o trabalho no Sistema Único de Saúde (SUS). In: Moysés ST, Kriger L, Moysés SJ. (Orgs.). Saúde bucal das famílias: trabalhando com evidências. São Paulo: Artes Médicas; 2008. p. 277-90.

13. Morita MC, Haddad AE. A concepção pedagógica e as Diretrizes Curriculares Nacionais: interface da área da educação e da saúde na perspectiva da formação e do trabalho das Equipes da Saúde da Família. In: Moysés ST, Kriger L, Moysés SJ. (Orgs.). Saúde bucal das famílias: trabalhando com evidências. São Paulo: Artes Médicas; 2008. 
p. 268-76.

14. Silva EVM. A formação de profissionais da saúde em sintonia com o SUS: currículo integrado e interdisciplinar. Núcleo de Gestão do Trabalho e Educação na Saúde do CONASEMS. Brasília: Ministério da Saúde, 2008.

15. Albuquerque VS, Batista RS, Tanji S, Moco ETSM. Currículos disciplinares da área da saúde: ensaio sobre saber e poder. Interface Comunic, Saúde, Educ 2009;13(31):261-72.

16. Werneck MAF, Senna MIB, Drumond MM, Lucas SD. Nem tudo é estágio: contribuições para o debate. Ciênc Saúde Colet. 2010; 15(1):221-31.

17. Zilbovicius C, Araujo ME, Botazzo C, Frias AC, Junqueira SR, Junqueira CR. A paradigm shift in predoctoral dental curricular in Brazil: evaluating the process of change. J Dent Educ. 2011;75(4):557-64.

18. Piskorowski WA, Fitzgerald M, Mastey J, Krell RE. Development of a sustainable community-based dental education program. J Dent Educ. 2011;75(8):1038-43.

19. González RD, Almeida MJ. Integralidade da saúde: norteando mudanças na graduação dos novos profissionais. Ciênc Saúde Colet. 2010;15(3):757-62.

20. Missaka H, Ribeiro VMB. A preceptoria na formação médica: subsídios para integrar teoria e prática na formação profissional - o que dizem os trabalhos nos congressos brasileiros de educação médica. Rev Bras Educ Méd. 2011;35(3):303-10.

21. Fonseca EP. The national curriculum guidelines and training of Brazilian dentists. Manag Prim Health Care. 2012;3(2):158-78.

22. Trajman A, Assunção N, Venturi M, Tobias D, Toschi W, Brant V. Student supervision in primary care clinics in the Rio de Janeiro city health department: opinions by healthcare professionals. Rev Bras Educ Méd. 2009; 33(1):24-32.

\section{Correspondência para:}

Rebeca Valeska Soares Pereira

e-mail: rebecavaleska@gmail.com

Rua Alcebíades Gonçalves da Rocha, 214 -

Santa Cruz

58417-335 Campina Grande/PB 